\title{
Role of long noncoding RNA taurine- upregulated gene 1 in cancers
}

\author{
Miao Da ${ }^{2}$, Jing Zhuang ${ }^{3}$, Yani Zhou ${ }^{4}$, Quan Qi ${ }^{5}$ and Shuwen Han ${ }^{1 *}$ (D)
}

\begin{abstract}
Long non-coding RNAs (IncRNAs) are a group of non-protein coding RNAs with a length of more than $200 \mathrm{bp}$. The IncRNA taurine up-regulated gene 1 (TUG1) is abnormally expressed in many human malignant cancers, where it acts as a competitive endogenous RNA (ceRNA), regulating gene expression by specifically sponging its corresponding microRNAs. In the present review, we summarised the current understanding of the role of IncRNA TUG1 in cancer cell proliferation, metastasis, angiogenesis, chemotherapeutic drug resistance, radiosensitivity, cell regulation, and cell glycolysis, as well as highlighting its potential application as a clinical biomarker or therapeutic target for malignant cancer. This review provides the basis for new research directions for IncRNA TUG1 in cancer prevention, diagnosis, and treatment.
\end{abstract}

Keywords: Long non-coding RNA (InCRNA), Taurine-upregulated gene 1 (TUG1), Cancer, Competitive endogenous RNA (ceRNA), Biomarker

\section{Introduction}

Cancer is the second leading cause of death in the United States, with about 600,000 deaths each year (Farhad et al. 2020). Cancer epidemiology is evolving as a result of altered risk factor patterns, changes in disease classification, improved testing and treatment, and demographic changes, including aging, population growth, and migration (Farhad et al. 2020). Cancer remains a disease with high morbidity and mortality, and represents a serious threat to human health (Claudia et al. 2020). There are various signal pathways and molecules involved in the progression, invasion, and metastasis of cancer cells. However, the molecular mechanisms underlying cancer are complex, and have not yet been fully explained. Therefore, it remains important to explore the molecular mechanisms of cancer, particularly regarding novel diagnostic and therapeutic strategies.

\footnotetext{
*Correspondence: ww@hzhospital.com

1 Department of Oncology, Huzhou Central Hospital, Affiliated Central Hospital Huzhou University, No. 1558, Sanhuan North Road, Wuxing, Huzhou, Zhejiang, People's Republic of China

Full list of author information is available at the end of the article
}

With the rapid development of genomics technology, the important role of non-coding RNAs (ncRNAs) has been clarified in the processes of growth, development, and disease (Mitchell and Rinn 2012). Thousands of ncRNAs have been identified, including various small RNAs (such as microRNA (miRNA), small nuclear/ nucleolar RNA, and piwi-interacting RNA), and a more uneven class of long non-coding RNAs (lncRNAs) (Taft et al. 2010). IncRNAs are a class of non-coding RNA molecules with a length of larger than 200 nt that have a mRNA-like structure, but do not encode a protein (Yihui et al. 2020). lncRNAs participate in embryogenesis, angiogenesis, and cancer progression by exerting epigenetic changes in many processes, including inactivation of $\mathrm{X}$ chromatin, regulation of the function of key metabolic genes, cell cycle control, and cell differentiation (Yao et al. 2019; Ding et al. 2020). Mounting evidence shows that lncRNAs are closely related to a variety of malignant cancers, and can play the role of oncogene or oncogene suppressor in different cancer types (Sisi et al. 2021; Miaomiao et al. 2021). For example, lncRNA MALAT1 promoted the progression of gastric cancer by inhibiting autophagy flux and 
inducing fibroblast activation (Zhenqiang et al. 2021). In renal cell carcinoma, HOTAIR and androgen receptors cooperatively increased GLI2 transcription to promote tumor angiogenesis and cancer stem cell property (Ji-Yu et al. 2021). Metastasis-associated protein 2 (MTA2) regulated by small nucleolar RNA host gene 5 (SNHG5) played an important role in the progression of oesophageal squamous cell carcinoma (Sisi et al. 2021).

Taurine upregulated gene 1 (TUG1), also known as TI$227 \mathrm{H}$, LINC00080 and NCRNA00080, is located on the human chromosome 22 autosomal long arm 1 region 2 sub-band (22 q12.2), with a total length of about $7.1 \mathrm{~kb}$ (Tang et al. 2018). This molecule was first found in neonatal mouse retinal cells, where it promoted the development of the retina (Young et al. 2005). This lncRNA interacts with the polycomb repressive complex and plays a role in the epigenetic regulation of transcription. Recently, lncRNA TUG1 research has mainly been focused on cancer, and lncRNA TUG1 can regulate the development of cancers (Yihui et al. 2020). lncRNA TUG1 is differentially expressed in cancers, and can affect the proliferation and apoptosis of cancer cells. The expression of lncRNA TUG1 is closely related to the prognosis of cancer patients (Ding et al. 2020). lncRNA TUG1 is thought to be involved in carcinogenesis and development mainly through competitive binding with miRNAs, regulation of cyclin-dependent kinase inhibitors, and effects on cancer proliferation and apoptosis (Young et al. 2005; Xiong et al. 2018) miRNAs are short RNAs that regulate a variety of physiological and biological processes in eukaryotic cells (Xia et al. 2020). miRNAs can bind to the $3^{\prime}$ untranslated region ( $3^{\prime}$-UTR) of the target gene mRNA, thus promoting mRNA cleavage or inhibiting mRNA translation (Li-Jun et al. 2017). Abnormal expression of lncRNA TUG1 affected the proliferation, apoptosis, and invasion of a variety of cancers, including bladder urothelial carcinoma, osteosarcoma, non-small cell lung cancer, and oesophageal squamous cell carcinoma, suggesting that lncRNA TUG1 may be used as a diagnostic marker or therapeutic target (Jun et al. 2016a; Qun et al. 2020).

In the present review, we evaluated the molecular mechanisms and clinical significance of lncRNA TUG1 in different cancer types. We summarized the research progress of lncRNA TUG1 in cancer cell proliferation, metastasis, angiogenesis, chemotherapeutic drug resistance, radiosensitivity, cell regulation, cell glycolysis, and its potential application as a clinical biomarker or therapeutic target for malignant cancer. A detailed summary of the review strategy is shown in Fig. 1. From Cancer Cell Line Encyclopedia (CCLE) database, Fig. 2 shows the expression of TUG1 in different cancers.

\section{Function of IncRNA TUG 1 in cancers}

\section{IncRNA TUG1 regulates cancer cell proliferation}

lncRNA TUG1 promoted cell proliferation in the digestive system. IncRNA TUG1 promoted cell growth, proliferation, and invasion, and induced apoptosis of oral squamous cell carcinoma cells by targeting the Wnt/Bcatenin signalling pathway (Shuang et al. 2017). lncRNA TUG1 promoted the progression of nasopharyngeal carcinoma by enabling miR-384 to inhibit the epithelialmesenchymal transformation (EMT) (Wei et al. 2019). lncRNA TUG1 promoted the proliferation and invasion of oesophageal squamous cell carcinoma cells by regulating the expression of cell division cycle 42 (CDC42) through miR-498 (Zhifeng et al. 2020). IncRNA TUG1/ miR-29c axis promoted the growth and migration of pancreatic cancer cells in vivo and in vitro (Yebin et al. 2018). lncRNA TUG1/miRNA-299-3p axis promoted the malignant progression of pancreatic cancer by inhibiting the Notch1 pathway (Ke and Lianfeng 2020).

IncRNA TUG1 was also found in the urinary system. lncRNA TUG1 promoted renal cell carcinoma formation via the miR-299-3p/vascular endothelial growth factor (VEGF) axis (Yunsheng et al. 2019). IncRNA TUG1 expression by miR142-mediated zinc finger E-box binding homeobox 2 (ZEB2), through inactivating the Wnt/ $\beta$ catenin pathway, promoted the proliferation of bladder cancer cells and induced apoptosis (Qian et al. 2017). Down-regulation of lncRNA TUG1 inhibited the development and progression of prostate cancer by regulating the microRNA496/wnt/ $\beta$-catenin pathway (Gang et al. 2020).

lncRNA TUG1 had also been found in other systems. Direct IncRNA TUG1 promoted the proliferation and invasion of glioma cells, and promoted apoptosis (Zhao et al. 2018). lncRNA TUG1 inhibited glioma cell proliferation (Jun et al. 2016a). In addition, IncRNA TUG1 acted as miR-26a sponge to up-regulate the expression of phosphatase and tensin homolog (PTEN), and inhibited the development of glioma (Jun et al. 2016b). lncRNA TUG1 was up-regulated in acute myeloid leukemia (AML) patients and cells, and lncRNA TUG1 promoted the proliferation and glycolysis of AML cells by targeting miR-185 (Weide et al. 2020). lncRNA TUG1 promoted the proliferation of AML cells and increased the rate of apoptosis (Jun et al. 2018a). IncRNA TUG1 promoted the proliferation and invasion of osteosarcoma cells through sponging miR-153 (Wang Heping and Yanzhang 2018).

The expression of IncRNA TUG1 was down-regulated in non-small cell lung cancer (Pei-Chin et al. 2016). IncRNA TUG1 inhibited the proliferation of non-small cell lung cancer cells (Pei-Chin et al. 2016). The effect of p53-regulated lncRNA TUG1 on the proliferation of non-small cell lung cancer cells was partly 

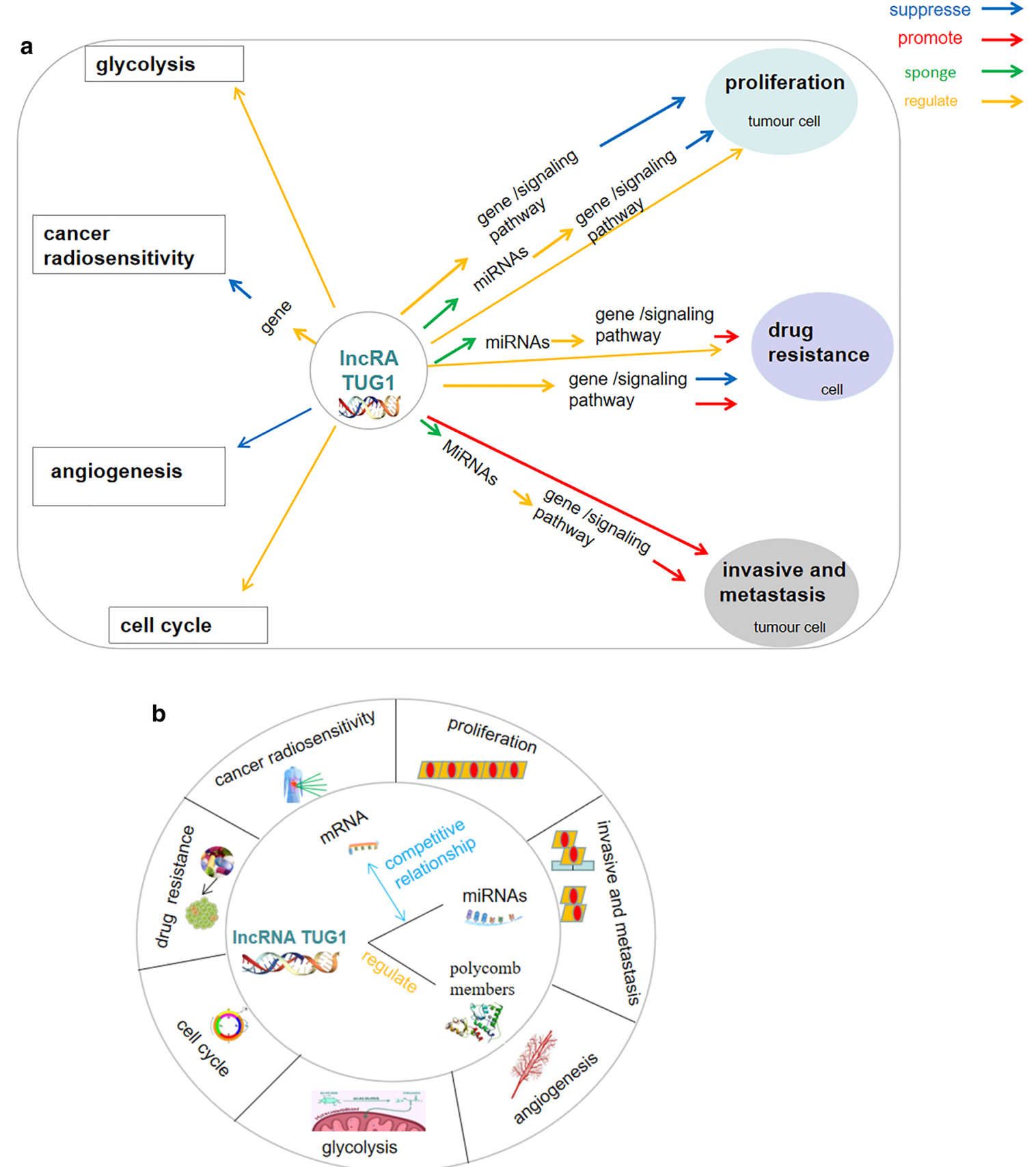

Fig. 1 Diagram showing review outline. The present review summarises the roles of IncRNA TUG1 in the regulation of cancer cell proliferation, metastasis, angiogenesis, chemotherapeutic drug resistance, radiosensitivity, cell regulation, and cell glycolysis

exerted through epigenetic regulation of homeobox B7 (HOXB7).(Zhang et al. 2014) lncRNA TUG1 inhibited the proliferation non-small cell lung cancer (Zhang et al. 2014). lncRNA TUG1 promoted the proliferation of MCF-7 breast cancer cells by inhibiting microRNA-9 (Xiao-Bo and Guo-Sheng 2016).
A summary of the regulation of cancer cell proliferation by lncRNA TUG1 in a variety of cancers is presented in Fig. 3. IncRNA TUG1 affected proliferation by regulating wnt $/ \beta$-catenin signal pathway, miR-384, miR-498, miR-29c, miR-299-3p, or microRNA-9, in oral squamous cell carcinoma, nasopharyngeal carcinoma,esophageal 


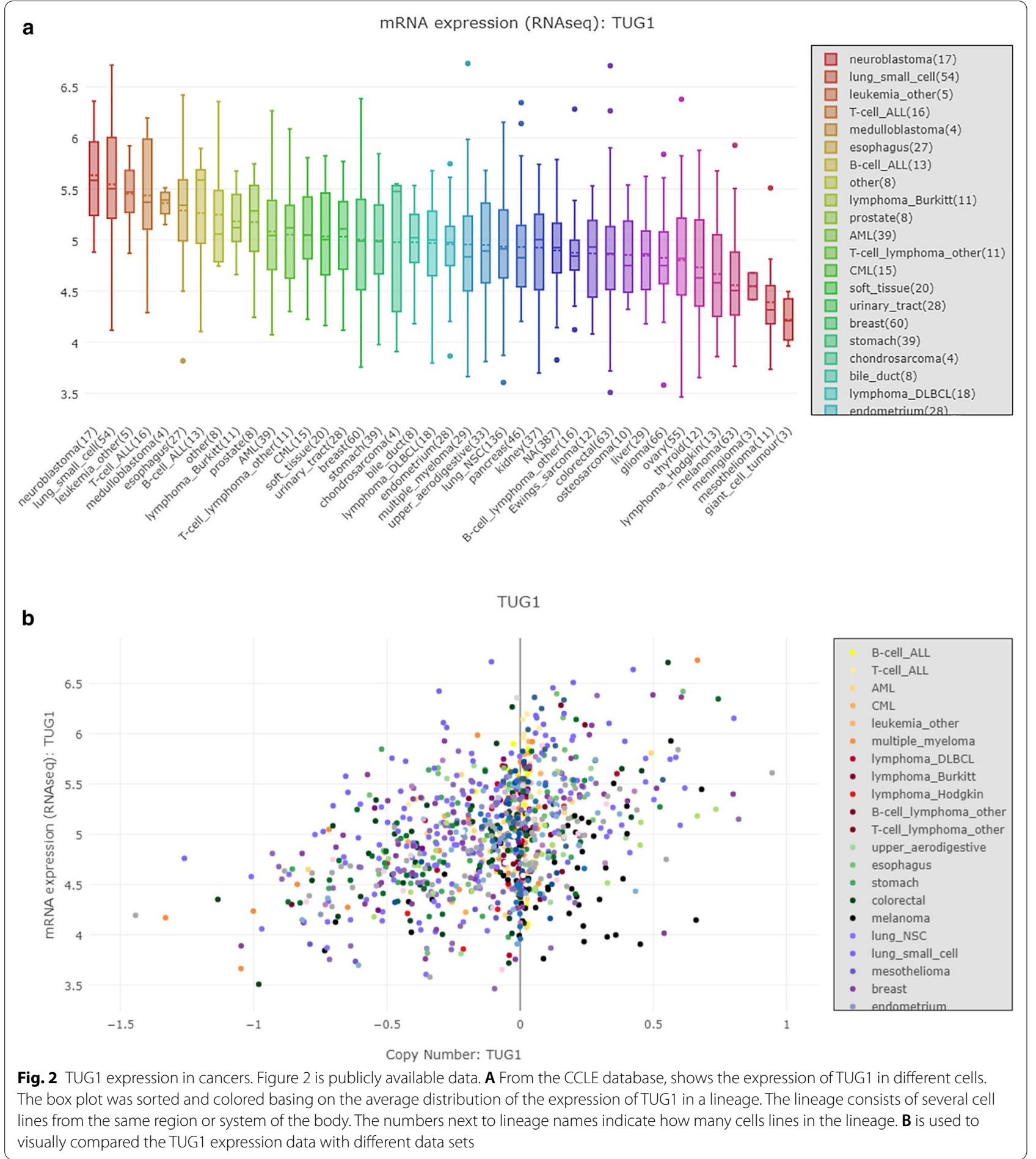

squamous cell carcinoma, pancreatic cancer, breast cancer, respectively. IncRNA TUG1 affected proliferation by $\mathrm{miR}-299-3 \mathrm{p} / \mathrm{VEGF}$ pathway, ZEB2/miR-142/wnt/ $\beta$ catenin pathway, microRNA496/wnt/ $\beta$-catenin pathway in renal cell carcinoma, bladder cancer, prostate cancer, respectively. IncRNA TUG1 affected proliferation by directly regulation, internal pathways mediated by caspase- 3 and-9, anti-apoptotic pathways mediated by Bcl2, or miR-26a sponging PTEN in glioma. lncRNA TUG1 affected proliferation by targeting miR-185 or directly 


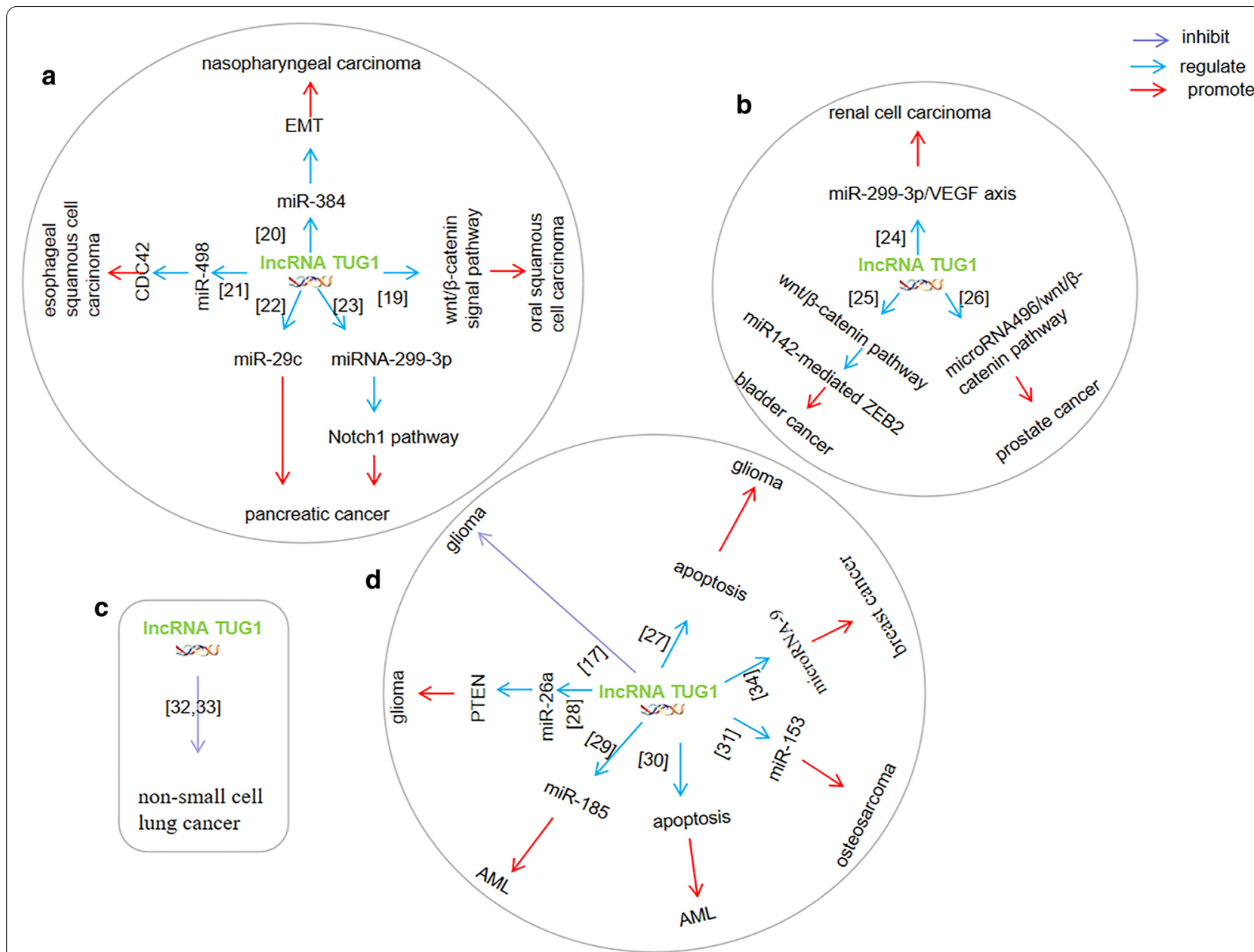

Fig. 3 InCRNA TUG1 regulates cancer cells proliferation of a variety of cancers. A network map illustrating the regulation of proliferation by IncRNA TUG1 in a variety of cancers, including oral cancer, oesophageal cancer, and pancreatic cancer. The pie charts labelled A, B, C and D represent cancers of the digestive system, cancers of urinary system, non-small cell lung cancer and cancers of other systems, respectively

regulating in AML. IncRNA TUG1 affected proliferation through miR-153 in osteosarcoma. However, the expression of lncRNA TUG1 was down-regulated in non-small cell lung cancer. IncRNA TUG1 inhibited proliferation of non-small cell lung cancer. Interestingly, the expression of lncRNA TUG1 in lung cancer was very special. At present, the research objects of literature reports on lung cancer are mainly from China. It was possible that the biological behavior of different tumors affected the expression of lncRNA TUG1. This situation deserves further study.

\section{IncRNA TUG1 regulates cancer invasion and metastasis}

Up-regulation of lncRNA TUG1 expression exerts a carcinogenic role by promoting the migration, and invasion of laryngeal cancer cells, and by inhibiting apoptosis (Zhang et al. 2018). lncRNA TUG1 participated in the development of laryngeal carcinoma via inhibiting the activation of RhoA/rho associated coiled-coil containing protein kinase (ROCK)/matrix metalloproteinase (MMPs) signalling pathway by miR-145-5p (Shenfa et al. 2019). Up-regulation of lncRNA TUG1 promoted the proliferation and migration of esophageal squamous cell carcinoma (Xu et al. 2015). lncRNA TUG1 acts as ceRNA by adjusting the miR-1294/PLK1 axis (Mingzhu et al. 2020), targeting miR-498 to induce XBP1 expression (Jin et al. 2020), or regulating the $\mathrm{Wnt} / \beta$-catenin pathway through the miR-148A-3p/mcl-1 axis (Yin et al. 2020) to promote growth and metastasis of oesophageal squamous cell carcinoma cells. IncRNA TUG1 promoted the proliferation and invasion of gastric cancer cells by negatively modulating miRNA-145-5p (Kewei et al. 2017). Overexpression of lncRNA TUG1 contributed to the proliferation and migration of colon cancer cells (HuiYuan et al. 2016). lncRNA TUG1 also regulates invasion and metastasis through other mechanisms, such as via 
miR-145-5p/TRPC6 (Xiaoqiang et al. 2021), the miR26a-5p/matrix metalloproteinases-14 (MMP-14)/p38 mitogen-activated protein kinase (p38MAPK)/HSP27 (heat shock protein 27) axis (Lei et al. 2019) the TUG1/ Twist1/EMT signalling pathway, (Shen et al. 2020), or by affecting the EMT (Liang et al. 2016) to promote proliferation and metastasis in colorectal cancer cells. The lncRNA TUG1/enhancer of zeste homolog 2 (EZH2) axis promoted the proliferation, migration, and EMT phenotype of pancreatic cancer cells by sponging miR-382 (Liang et al. 2017).

To sum up, IncRNA TUG1 promoting invasion and metastasis by some signal pathways, including RhoA/ ROCK/MMPs axis, miR-1294/PLK1 axis, miR-498/XBP1 axis, $\mathrm{miR}-148 \mathrm{~A}-3 \mathrm{p} / \mathrm{mcl}-1 / \mathrm{Wnt} / \beta$-catenin axis, miR145-5p/TRPC6 axis, miR-26a-5p/MMP-14/p38MAPK/ HSP27 aix, or Twist1/EMT axis, and some molecule, including miR-145-5p, EMT, EZH2, or miR-382 in cancers.

lncRNA TUG1 promoted the development of osteosarcoma through runt-related transcription factor 2 (RUNX2) (Kunkun and Yan 2019). IncRNA TUG1 regulated the proliferation and invasion of osteosarcoma through a variety of different miRNA, such as hypoxia inducible factor- $1 \alpha$ mediated by miR-143p (Yu Xiao and Lei 2019), sponging miR-9-5p and regulation of POU2F1 expression (Chu-Hai et al. 2016), miR-212-3p/forkhead box A1 (FOXA1) axis (Chuhai et al. 2018), miR-140-5p/ PFN2 axis (Zhao et al. 2019), inhibition of microRNA212-3p expression (Heng et al. 2018), miR-335-5p on Rho-associated coiled-coil containing protein kinase 1 (ROCK1) expression and ROCK1-mediated expression (Yong et al. 2017), or sponging of miR-132-3p and up-regulation of sex determining region Y-box 4 (SOX4) expression (Gang et al. 2018). There was also a positive and negative feedback regulation mode for lncRNA TUG1 in osteosarcoma. Forkhead Box M1 (FOXM1) up-regulated the expression of lncRNA TUG1 in osteosarcoma cells (Yang et al. 2018a). lncRNA TUG1 promoted the proliferation, migration, and invasion of osteosarcoma through competitively sponging of miR-219a-5p, resulting in the up-regulation of phosphatidylinositol-4, 5-bisphosphate 3-kinase catalytic subunit alpha (PIK3CA) and the activation of the protein kinase B (AKT) signal pathway (Yang et al. 2018a). In addition, the activation of the AKT pathway promoted the expression of lncRNA TUG1 by upregulating the expression of FOXM1, forming a positive feedback loop in osteosarcoma (Yang et al. 2018a).

To sum up, IncRNA TUG1 promoted invasion and metastasis by some molecule, including miR-143p, miR-9-5p, miR-212-3p, miR-219a-5p, miR-140-5p, miR212-3p, miR-335-5p, miR-132-3p, SOX4, RUNX2, PFN2, ROCK1,FOXA1, or POU2F1 in osteosarcoma.
lncRNA TUG1 promoted the growth and metastasis of cholangiocarcinoma cells by inhibiting miR-29a (Yuan et al. 2020). lncRNA TUG1 sponging of miR-145 promoted intrahepatic cholangiocarcinoma progression, and regulated glutamine metabolism through the signal transducer and activator of transcription 3 (STAT3)/ GDH axis (Bing et al. 2017). lncRNA TUG1 promoted hepatoma cell proliferation, migration and invasion, inhibited apoptosis, and up-regulated AURKA expression in hepatocellular carcinoma (Peng et al. 2018a). IncRNA TUG1 interacted with a variety of miRNAs to promote hepatoma cell migration, and invasion, such as miR-144 through activation of the Janus kinase 2 (JAK2)/STAT3 pathways (Jun et al. 2018b), the miR-216b-5p/DLX2 axis (Qun et al. 2020), downregulation of miR-142-3p (Chuan et al. 2018), or the miR-29c-3p/ collagen type 1 alpha 1 (COL1A1) axis (Wei et al. 2020).

lncRNA TUG1 knockout promoted cell growth by promoting cell cycle progression and regulating the expression of cyclinD1 and CDK4 (Fan et al. 2017). IncRNA TUG1 suppressed miR-196A (Yang et al. 2018b), regulated the MIR-31-5p/flotillin 1 (FLOT1) axis (Dong et al. 2020), or regulated yes-associated protein (YAP) (Shan et al. 2018) to promote the proliferation and migration of renal cell carcinoma. The upregulation of lncRNA TUG1 promoted the proliferation, migration, and invasion of bladder cancer cells by inhibiting miR-29c (Peng et al. 2018b). IncRNA TUG1, as the ceRNA of miR-26a, promoted the progression of prostate cancer (Bin et al. 2018). IncRNA TUG1 accelerated the progression of prostate cancer by regulating the MIR-128-3p/YES1 axis (Hao et al. 2020).

Up-regulation of IncRNA TUG1 promoted the proliferation and migration of cervical cancer cells (Yingying et al. 2017). IncRNA TUG1 predicted a poor prognosis in epithelial ovarian cancer, promoted cell proliferation, and inhibited apoptosis (Tong-Huai et al. 2018). lncRNA TUG1 also regulated aurora kinase A (AURKA) (Tonghuai et al. 2018), or sponged miR-1299 by up-regulating notch receptor 3(NOTCH3) (Yuqing et al. 2020) to promote the proliferation and invasion of epithelial ovarian cancer cells. IncRNA TUG1 promoted the progression of ovarian cancer by targeting the mir-29b-3p/MDM2 axis (Xiaoqiu et al. 2020).

In AML patients, IncRNA TUG1 was associated with disease progression and poor prognosis, induced AML cell proliferation, and inhibited apoptosis by targeting AURKA (Xinfeng et al. 2018). lncRNA TUG1 also promoted the proliferation, migration, and invasion of AML cells by regulating miR-370-3p/MAPK1/ERK (Gang et al. 2019).

Figure 4 summarises the regulation of invasion and metastasis by lncRNA TUG1 in a variety of cancers. 
(See figure on next page.)

Fig. 4 IncRNA TUG1 regulates cancer invasion and metastasis in many different cancer types. A diagram showing the regulation of cancer invasion and metastasis by IncRNA TUG1 in different cancer types. The pie charts labelled $\mathbf{A}-\mathbf{Q}$ represent laryngeal squamous cell carcinoma, oesophageal cancer, non-small cell lung cancer, colorectal cancer, osteosarcoma, hepatocellular carcinoma, renal cell carcinoma, bladder cancer, prostate cancer, gastric cancer, cervical cancer, intrahepatic cholangiocarcinoma, ovarian cancer, acute myeloid leukemia, pancreatic cancer, breast cancer, and cholangiocarcinoma, respectively

\section{IncRNA TUG1 regulates cell cycle}

The progression of the cell cycle is controlled by the induction of cyclins and the activation of homologous cyclin-dependent kinases (Xiao et al. 2020). IncRNA TUG1 acts on amplified in breast cancer 1 (AIB1) to regulate the cell cycle in ovarian cancer (Li et al. 2017a). The expression of G1 phase related proteins was significantly changed ( $\mathrm{Li}$ et al. 2017a). In tongue squamous cell carcinoma, down-regulation of lncRNA TUG1 inhibited cell proliferation, and silencing of lncRNA TUG1 regulated the progression of the cell cycle ( $\mathrm{Li}$ et al. 2017b). TUG1 knockout blocked cell cycle, accelerated apoptosis and inhibitted the proliferation of pancreatic cancer cells (Hui Bingqing and Yetao 2019). Knocking out the TUG1 reduced that enhancer of zeste homolog 2 (EZH2) binded to the promoter regions of Rho family GTPase 3 (RND3) and metallothionein 2A (MT2A) (Hui Bingqing and Yetao 2019).

\section{IncRNA TUG1 regulates cellular glycolysis}

Glycolysis is an oxygen-independent metabolic pathway. In this process, glucose is converted to pyruvate, which then produces lactic acid (Ozcan Selahattin et al. 2020). Glycolysis reflects a change in the energy metabolism of cancer cells. In the presence of oxygen, malignant cells have higher glycolysis rates than normal cells, which is known as the "Warburg Effect" (Ozcan Selahattin et al. 2020; Bensinger and Christofk 2012). lncRNA TUG1 knockout inhibited glucose consumption, lactic acid production, and reduced the cell viability of osteosarcoma cells. Overexpression of lncRNA TUG1 increased cell viability, while 2-deoxy-D-glucose (2-DG) could attenuate this increase. The abnormal expression of lncRNA TUG1 significantly affected the expression of hexokinase-2 (HK2), which might be an important molecule through which lncRNA TUG1 affects glycolysis (Xiufu et al. 2018). HK2 gene knockout weakened the effect of lncRNA TUG1 overexpression on glycolysis in osteosarcoma cells (Xiufu et al. 2018). IncRNA TUG1 was up-regulated in AML patients and cells, and its knockout inhibited glycolysis in AML cells by targeting miR-185 (Weide et al. 2020).

\section{IncRNA TUG1 regulates cancer angiogenesis}

Angiogenesis is one of the prerequisites for active cancer progression. Under the regulation of hormones, including VEGF, angiogenesis plays a key role in the pathogenesis of ovarian cancer (Protopsaltis Nicholas et al. 2019). lncRNA TUG1 induced the expression of VEGF, cancer growth factor- $\alpha$, and angiopoietin-1 in vascular endothelial cells through leucine-rich $\alpha-2$ glycoprotein-1 (LRG1) (Mingjun et al. 2019). Knockout of lncRNA TUG1 inhibited angiogenesis in ovarian cancer by regulating LRG1 (Mingjun et al. 2019).

\section{IncRNA TUG1 regulates resistance and sensitivity to chemotherapeutic drugs}

Chemotherapy resistance remains the limiting factor in the treatment of cancer (Daniela and Rosario 2020). lncRNA TUG1 inhibited the expression of PDCD4 through epigenetic pathways (Caihui et al. 2018), or by up-regulating nuclear factor (erythroid-derived 2)-like 2 (Nrf2) (Zhenghua et al. 2019), to make confer cisplatin resistance in oesophageal squamous cell carcinoma. lncRNA TUG1 regulated CCND2, through EZH2-related miR-194-5p silencing, to promote the growth of bladder cancer cells and confer cisplatin resistance (Gan et al. 2019). Low expression of lncRNA TUG1 enhanced the sensitivity of cervical cancer to cisplatin by activating the MAPK pathway (Xuemin et al. 2019). Down-regulation of lncRNA TUG1 inhibited cisplatin resistance in drug-resistant tongue squamous cell carcinoma cells, by mediating miR-133b and cysteine-X-cysteine chemokine receptor 4 (CXCR4) (Ke et al. 2020). IncRNA TUG1 knockout can induce apoptosis by inhibiting MET/Akt signalling, thus reducing the resistance of osteosarcoma cells to cisplatin (Zhou Qiang and Yuan 2020).

Up-regulation of lncRNA TUG1 expression in bladder urothelial carcinoma inducing by transcription factor Nrf2 promoted cancer progression and adriamycin resistance (Zhulei et al. 2019). Polydatin inhibition of Akt signalling, mediated by lncRNA TUG1, suppressed the proliferation of doxorubicin-resistant osteosarcoma and promoted its apoptosis (Tongzhou et al. 2019). lncRNA TUG1 induced autophagy of ovarian cancer cells by targeting miR-29b-3p, which lead to drug resistance to paclitaxel (Lize et al. 2020). 


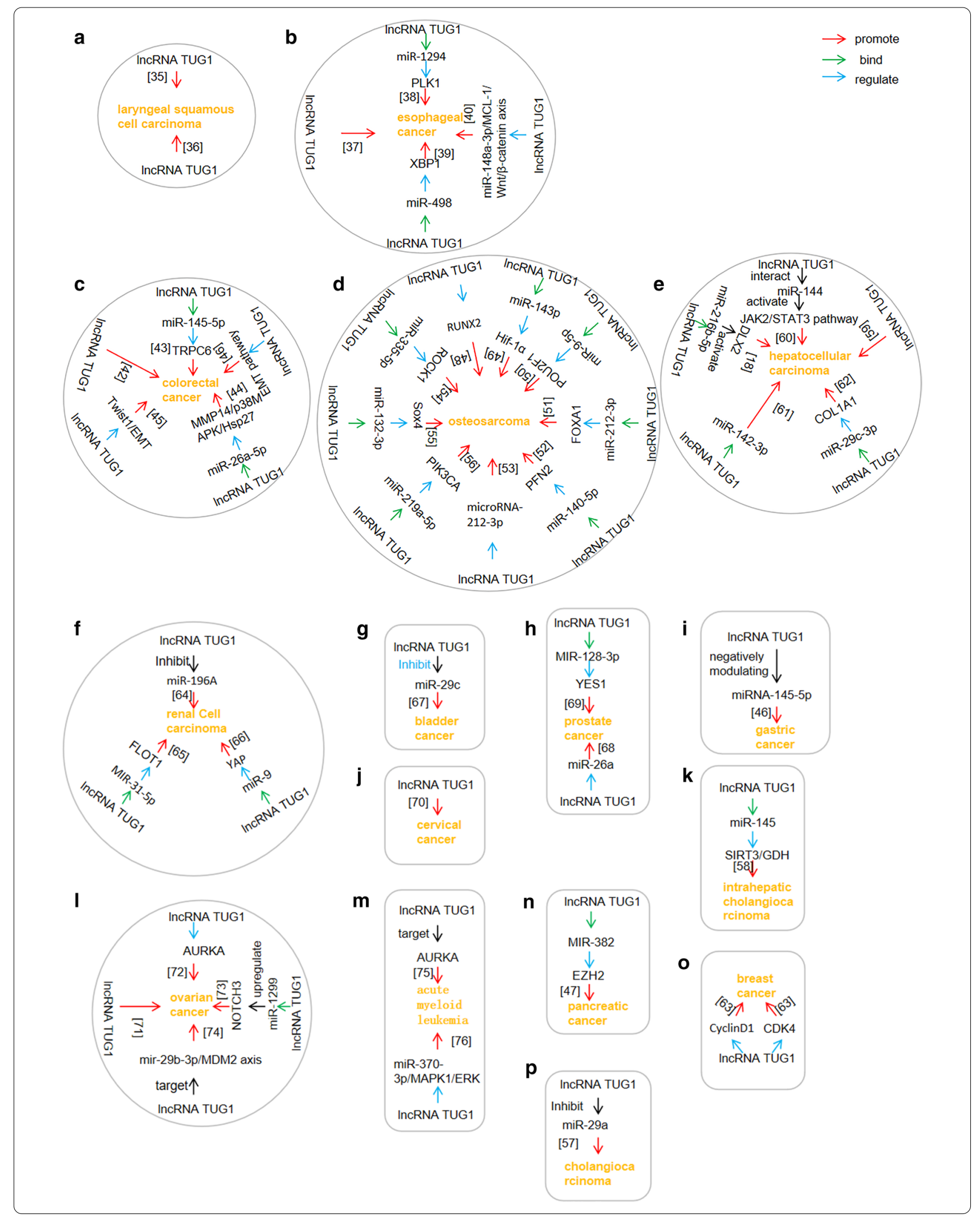




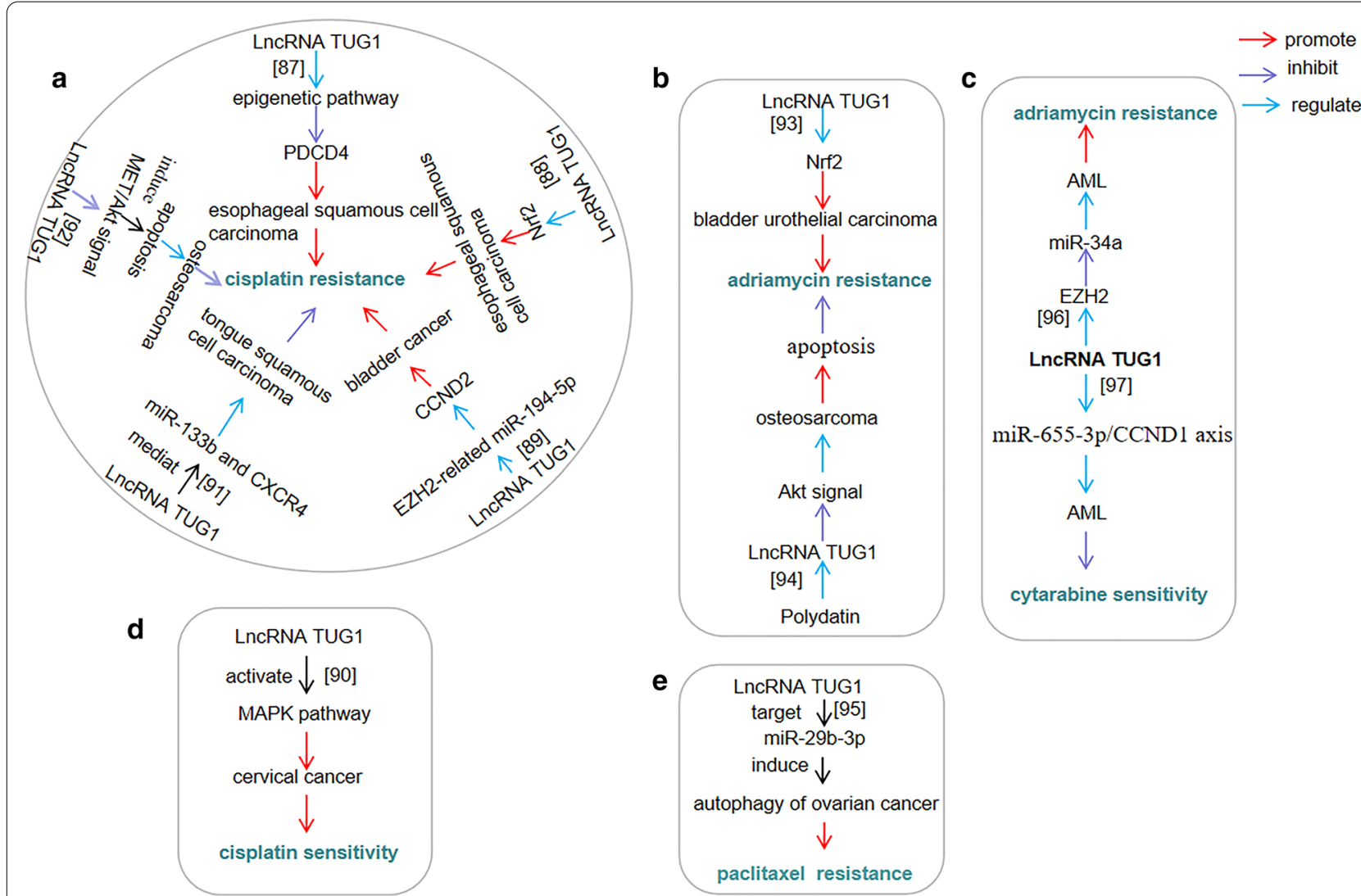

Fig. 5 IncRNA TUG1 regulates resistance and sensitivity to chemotherapeutic drugs. A diagram showing the regulation of resistance and sensitivity to chemotherapeutic drugs by IncRNA TUG1 in different cancer types. The pie charts labelled $\mathbf{A}, \mathbf{B}, \mathbf{C}, \mathbf{D}$, and $\mathbf{E}$ represent cisplatin resistance,

adriamycin resistance, cytarabine sensitivity, cisplatin sensitivity, and paclitaxel resistance, respectively

lncRNA TUG1 enhanced adriamycin resistance in AML by inhibiting the expression of miR-34a through EZH2 epigenetically ( $\mathrm{Li}$ et al. 2019). IncRNA TUG1 reduced the sensitivity of AML cells to cytarabine by regulating the miR-655-3p/cyclin D1 (CCND1) axis (Zhang et al. 2020).

Figure 5 shows a summary of the regulation of resistance and sensitivity to chemotherapeutic drugs in different cancers by lncRNA TUG1.

\section{IncRNA TUG1 regulates radiosensitivity}

Radiotherapy is an effective strategy to prevent cancer metastasis. However, radiation resistance in cancer can limit the efficiency of radiotherapy (He Pan and Yong-Qiang 2020). The expression of lncRNA TUG1 was up-regulated in bladder cancer tissues and cell lines. The down-regulation of lncRNA TUG1 enhanced the radiosensitivity of bladder cancer cells by inhibiting the expression of high mobility group box-1 protein (HMGB1) (Jiang Huijuan and Xigang 2017). Knockout of lncRNA TUG1 enhanced the radiosensitivity of prostate cancer through the IncRNA TUG1/miR-139-5p/ structural maintenance of chromosomes protein 1A (SMC1A) axis (Dianhui et al. 2020). lncRNA TUG1 upregulated the expression of MET through sponging miR144-3p, which activated the AKT signalling pathway and enhanced the radiosensitivity of oesophageal carcinoma (Pan et al. 2020).

\section{Clinical studies of IncRNA TUG1 in cancers}

Current clinical studies of lncRNA TUG1 are shown in Table 1. The high expression of lncRNA TUG1 was associated with chemotherapy resistance and poor prognosis in oesophageal squamous cell carcinoma (Lin et al. 2016). Conversely, the expression of lncRNA TUG1 in viral hepatitis $\mathrm{C}$ and viral hepatitis $\mathrm{C}$-associated hepatocellular carcinoma decreased (Mai et al. 2020). lncRNA TUG1 was used as a non-invasive, cost-effective, and complementary biomarker in viral hepatitis $\mathrm{C}$ and viral hepatitis C-associated hepatocellular carcinoma (Mai et al. 2020). The level of alpha-fetoprotein (AFP) in patients with nonhepatitis $\mathrm{B} /$ non-C hepatocellular carcinoma (NBNCHCC) was positively correlated with that of IncRNA TUG1, and the prognosis was poor (Lin et al. 2020). 


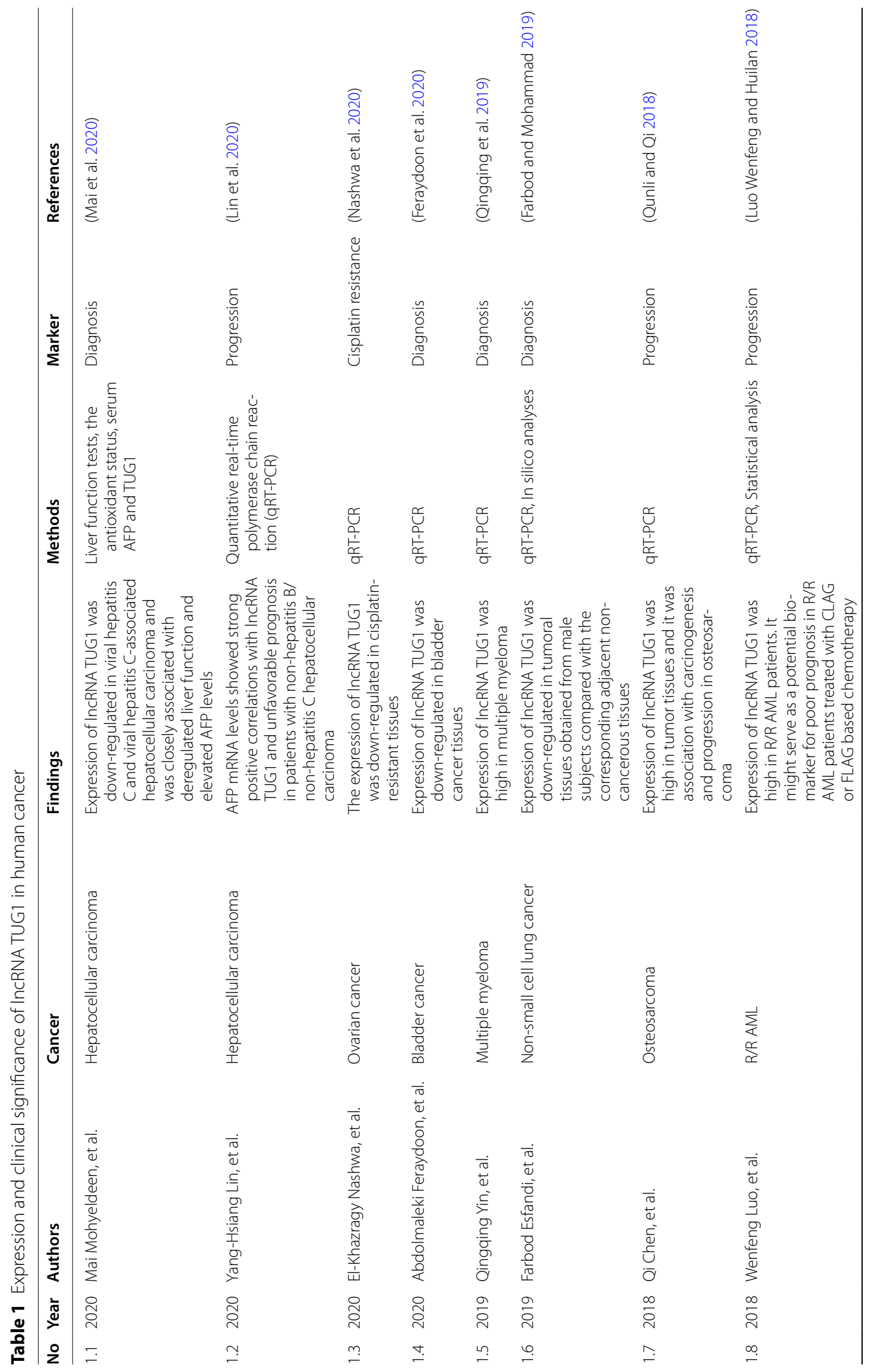




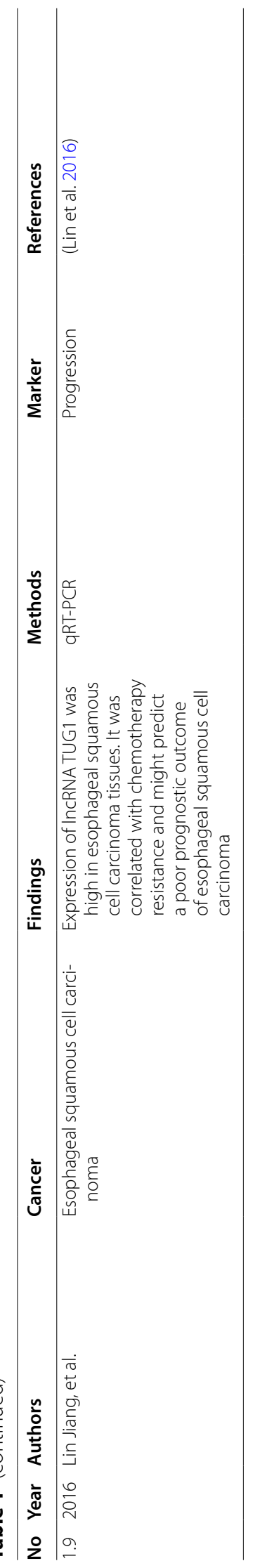


lncRNA TUG1 may be an effective prognostic marker of NBNC-HCC (Lin et al. 2020). The expression of lncRNA TUG1 in male lung cancer tissues was lower than that in the corresponding paracancerous tissue (Farbod and Mohammad 2019). The expression of IncRNA TUG1 was down-regulated and used as a diagnostic marker in bladder cancer tissues (Feraydoon et al. 2020). lncRNA TUG1 can be used to predict the resistance of ovarian cancer patients to cisplatin (Nashwa et al. 2020). Elevated lncRNA TUG1 levels was a potential biomarker for the diagnosis of multiple myeloma (Qingqing et al. 2019). The expression of IncRNA TUG1 in osteosarcoma was significantly higher than that in adjacent normal bone tissue (Qunli and Qi 2018). The up-regulation of lncRNA TUG1 expression was significantly correlated with larger tumour size and late stages of lymph node metastasis in patients with osteosarcoma (Qunli and Qi 2018). However, low expression levels of lncRNA TUG1 was an independent indicator of poor prognosis in patients with osteosarcoma (Qunli and Qi 2018). lncRNA TUG1 was highly expressed in refractory or relapsed acute myeloid leukaemia (R/R AML), and may be a potential biomarker of poor prognosis in patients with R/R AML treated with granulocyte colony-stimulating factor (G-CSF) (CLAG) or fludarabine combined with cytarabine and G-CSF (FLAG) chemotherapy (Luo Wenfeng and Huilan 2018).

Sum up, IncRNA TUG1 have been used as a biomarker in esophageal squamous cell carcinoma, viral hepatitis $\mathrm{C}$ and viral hepatitis $\mathrm{C}$-associated hepatocellular carcinoma, NBNC-HCC, lung cancer, bladder cancer, ovarian cancer, osteosarcoma and R/R AML.

\section{Discussion}

At present, the literature reviews on lncRNA TUG1 are mainly based on the classification of different cancers. In the present review, we have comprehensively analysed and classified the literature related to lncRNA TUG1 into its molecular mechanisms and clinical research categories. The molecular mechanisms were mainly summarized based on cancer cell proliferation, metastasis, angiogenesis, chemotherapeutic drug resistance, radiosensitivity, cell regulation, and cell glycolysis. At present, there are relatively fewer clinical studies on lncRNA TUG1, but existing studies suggest that IncRNA TUG1 may be an effective diagnostic or prognostic cancer biomarker.

lncRNAs can act as ceRNAs to inhibit the function of miRNAs by preventing the interaction between miRNAs and their target mRNAs, thus affecting the translation of protein-coding genes (Zhang et al. 2020). Fig. 6 shows the miRNAs that are targeted by lncRNA TUG1 as a ceRNA to regulate cancer growth, metastasis, angiogenesis, and chemotherapeutic drug resistance.
lncRNA TUG1 promoted the proliferation of cancers, such as oral squamous cell cancer, bladder cancer, and prostate cancer. However, it had an inhibitory effect on the proliferation of non-small cell lung cancer. TUG1 played different roles in different cancers. It might be related to cancer biological behavior and other related regulatory factors. lncRNA TUG1 promoted the migration, invasion and metastasis of laryngeal cancer, esophageal squamous cell cancer and gastric cancer. lncRNA TUG1 induced cycle arrest in pancreatic cancer cells and promoted apoptosis. The glycolysis process of acute myelogenous leukemia was affected by lncRNA TUG1 to promote cancer growth. It was also stimulative for angiogenesis of ovarian cancer. Therefore, lncRNA TUG1 played a role in cancer cell proliferation, migration, invasion, cell cycle, angiogenesis and glycolysis. In esophageal squamous cell cancer and bladder cancer, lncRNA TUG1 was involved in the occurrence of cisplatin resistance, and the development of azithromycin resistance in urothelial cancer of the bladder and acute myelogenous leukemia. Therefore, lncRNA TUG1 was involved in the process of chemotherapeutic drug resistance in different cancers. Although lncRNA TUG1 could reduce the radiosensitivity of bladder cancer, it could also enhance the radiosensitivity of esophageal cancer. This paradox suggested that lncRNA TUG1 had different effects on the radiosensitivity of different cancers. It might be related to cancer heterogeneity, cancer microenvironment or immune system.

lncRNA TUG1 can regulate a variety of signal pathways, especially the $\mathrm{Wnt} / \beta$-catenin pathway, during the development of cancer. As a highly conserved and tightly regulated signal pathway, Wnt/ $\beta$-catenin plays an important role in the regulation of embryonic development, cell proliferation, and differentiation (Zhiqiang et al. 2020). If any of the key proteins in this signalling pathway are mutated, resulting in abnormal signal activation, it may induce the development of cancer (Shuang et al. 2017). For example, activating the Wnt/ $\beta$-catenin signalling pathway regulated the invasion and proliferation of oesophageal squamous cell carcinoma, cervical cancer, bladder cancer, and colorectal cancer, and induced their epithelial cell transformation ( $\mathrm{Fu}$ Bing et al. 2020). In accordance, lncRNA TUG1 can regulate the growth, proliferation, and invasion of oral squamous cell carcinoma, bladder cancer, and prostate cancer through the $\mathrm{Wnt} / \beta$-catenin signalling pathway. The main components of Wnt signal pathway include secretory protein Wnt family, transmembrane receptor Frizzled family, Axin, $\beta$-Catenin, and transcription factor TCF/LEF family, etc. However, it is not clear which components are involved in the regulation of wnt signal 


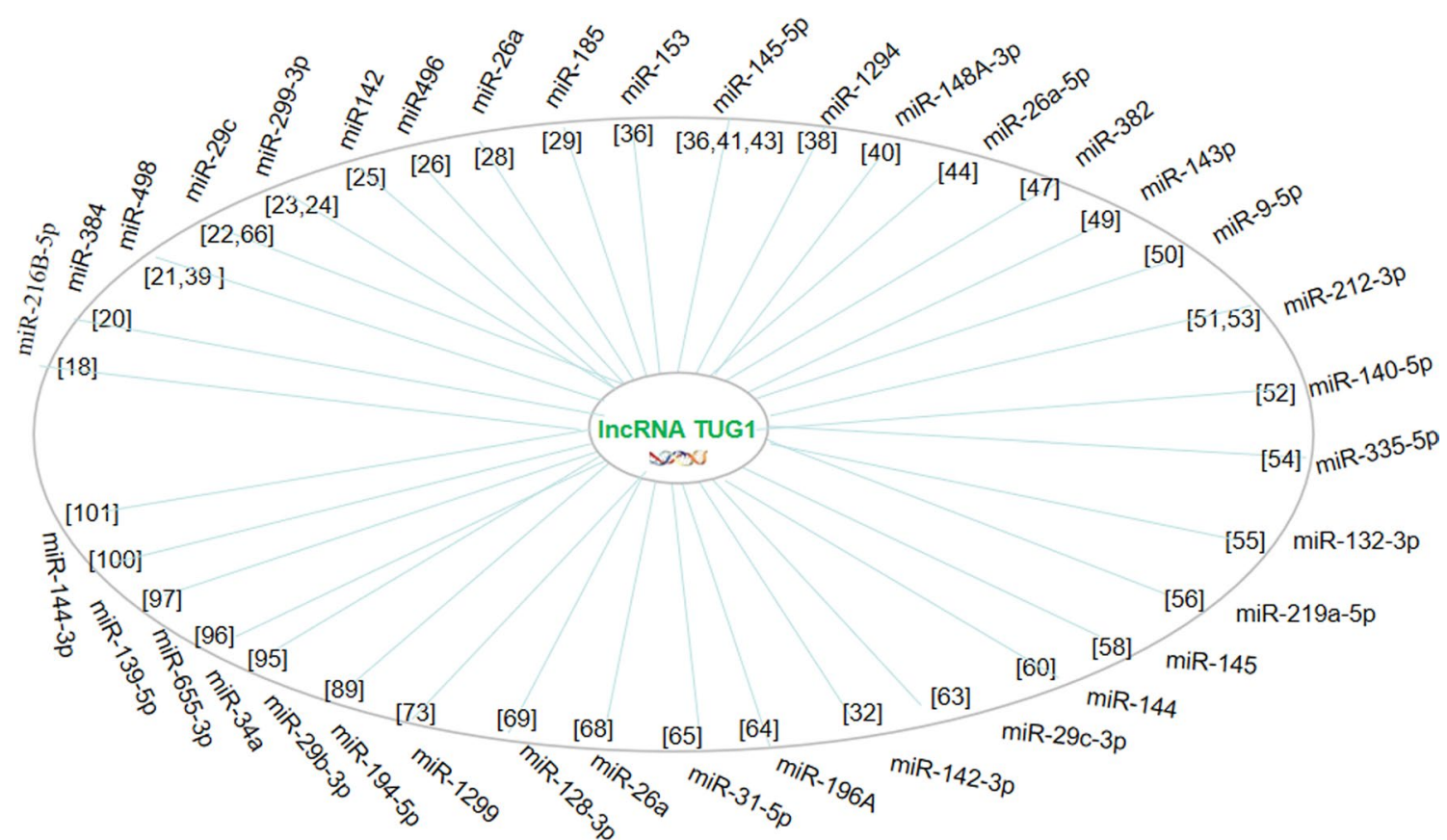

Fig. 6 IncRNA TUG1 acts as ceRNA to compete with miRNAs. A diagram summarising the activity of IncRNA TUG1 as a ceRNA to compete with miRNAs in different cancer types

pathway by lncRNA TUG1, which still need to be further explored.

In addition to Wnt signalling, lncRNA TUG1 can also regulate the development of cancers through other pathways. IncRNA TUG1 promoted the proliferation of oesophageal squamous cell carcinoma cells by regulating the miR-1294/PLK1 axis, either by inducing XBP1 expression via miR-498, regulating the $\mathrm{Wnt} / \beta$-catenin pathway via the miR-148A-3p/mcl-1 axis, or by direct up-regulation. IncRNA TUG1 promoted the proliferation and migration of colorectal cancer cells through the miR-145-5p/TRPC6 pathway, EMT pathway, miR26a-5p/MMP14/p38MAPK/Hsp27 axis, Twist1/EMT signal pathway, or by overexpression. IncRNA TUG1 promoted the proliferation, migration, and invasion of hepatoma cells by activating the JAK2/STAT3 pathway, upregulating the expression of AURKA, interacting with miR-216B-5p and inhibiting apoptosis by activating DLX2, or by down-regulating miR-142-3p to regulate the miR-29c-3p/COL1A1 axis. IncRNA TUG1 regulated downstream genes, including miR-143p, miR-9-5p, miR212-3p, miR-140-5p, microRNA-212-3p, miR-335-5p and miR-219a-5p, to participate in the proliferation and invasion of osteosarcoma cells. There are multiple regulatory pathways in each kind of tumor, but further molecular experimental studies are still needed to clarify the interactions and relationships between various pathways.

\section{Future research direction}

The functions of lncRNAs are diverse and complex. lncRNAs can regulate gene expression at different stages, steps, and levels, including epigenetically, transcriptionally, post-transcriptionally, and via miRNA. There are four main modes of action of lncRNA, denoted signal, decoy, guide, and scaffold. After decoy lncRNAs are transcribed, they bind to RNAs (ceRNA)/protein (transcription factor/transcriptional regulator), thus blocking the action of the RNA molecule. Currently, it has been established that lncRNA TUG1 acts as a decoy lncRNA to exert its role in cancers. However, future studies still need to explore other possible mechanisms of action for lncRNA TUG1. At present, the molecular and clinical studies on lncRNA TUG1 are in their infancy. Although studies suggest that lncRNA TUG1 mainly regulates miRNAs downstream, there remains a lack of specific types of molecular experiments to verify these. Further molecular experiments should be carried out to expand the role of lncRNA TUG1 in different cancer types, and to explore its role in body fluids. A more complete overview of the role of lncRNA TUG1 in regulating miRNA in different cancers could provide guidance for the diagnosis and treatment of cancers in future. In addition, established machine learning models based on a large number of sequencing data can predict the molecular regulatory network with regard to lncRNA TUG1 in cancers. This can provide a new direction for the future. 


\section{Conclusion}

It is a long and complicated process to explore the relation between lncRNA TUG1 and cancer. In the present review, we summarized the available literature to show that lncRNA TUG1 can regulate cancer cell proliferation, metastasis, angiogenesis, chemotherapeutic drug resistance, radiosensitivity, cell regulation, and cell glycolysis by regulating multiple molecular signalling pathways. This lncRNA could be used as a potential molecular target for cancer diagnosis and treatment in the future. The evaluation of the expression levels and functions of lncRNA TUG1 in cancer requires further research to provide a reference for accurate targeted therapy.

\begin{abstract}
Abbreviations
AKT: Activation of protein kinase B; AURKA: Aurora kinase A; AML: Acute myeloid leukemia; AIB1: Amplified in breast cancer 1; AFP: Alpha-fetoprotein; ceRNA: Competitive endogenous RNA; CDC42: Cell division cycle 42; COL1A1: Collagen type 1 alpha 1; CELF1: CUGBP and Elav-like family member 1; CXCR4: Cysteine-X-cysteine chemokine receptor 4; CCLE: Cancer Cell Line Encyclopedia; G-CSF: Granulocyte colony-stimulating factor; EMT: Epithelial-mesenchymal transformation; EZH2: Enhancer of zeste homolog 2; FLAG: Fludarabine combined with cytarabine and G-CSF; FOXA1: Forkhead box A1; FOXM1: Forkhead Box M1; FLOT1: Flflotillin 1; HSP27: Heat shock protein 27; HMGB1: High mobility group box 1 protein; HK2: Hexokinase-2; HOXB7: Homeobox B7; HOTAIR: Hox transcript antisense intergenic RNA; JAK2: Janus kinase 2; LRG1: Leucine-rich a-2-glycoprotein-1; IncRNAs: Long non-coding RNAs; MT2A: Metallothionein 2A; MiRNA: MicroRNA; MMPs: Matrix metalloproteinases; MMP-14: Matrix metalloproteinases-14; MAPK1: Mitogen-activated protein kinase 1; MTA2: Metastasis-associated protein 2; NOTCH3: Notch receptor 3; Nrf2: Nuclear factor (erythroid-derived 2)-like 2; NBNC-HCC: Non-hepatitis B / non-C hepatocellular carcinoma; PTEN: Phosphatase and tensin homolog; p38MAPK: P38 mitogen-activated protein kinase; PIK3CA: Phosphatidylinositol-4, 5-bisphosphate 3-kinase catalytic subunit alpha; R/R AML: Refractory or relapsed acute myeloid leukemia; RND3: Rho family GTPase 3; ROCK: Rho associated coiled-coil containing protein kinase; ROCK1: Rho-associated coiled-coilcontaining protein kinase 1; SOX4: Sex determining region Y-box 4; STAT3: Signal transducer and activator of transcription 3; SMC1A: Structural maintenance of chromosomes protein 1A; SHNG3: Small nucleolar RNA host gene 3; SNHG5: Small nucleolar RNA host gene 5; TUG1: Taurine up-regulated gene 1; VEGF: Vascular endothelial growth factor; YAP: Yes-associated protein; ZEB2: Zinc finger E-box binding homeobox 2; 2-DG: 2-Deoxy-D-glucose.
\end{abstract}

\section{Acknowledgements}

Not applicable.

\section{Authors' contributions}

All authors participated in the conception and design of the study. SH and MD conceived the study and prepared the manuscript. SH and MD designed and drew the network. JZ, YZ, and QQ reviewed and sorted the literature. All authors read and approved the final manuscript.

\section{Funding}

This work was supported by Zhejiang Provincial Natural Science Foundation (No. LQ20H160001) and Zhejiang Medical and Health Technology Projects (No. 2020RC117).

\section{Availability of data and materials}

Not applicable.

\section{Declarations}

Ethics approval and consent to participate

As no human subjects were recruited for this study, obtaining consent is not applicable.

\section{Consent for publication}

Not applicable.

\section{Competing interests}

The authors declare no conflicts.

\section{Author details}

${ }^{1}$ Department of Oncology, Huzhou Central Hospital, Affiliated Central Hospital Huzhou University, No. 1558, Sanhuan North Road, Wuxing, Huzhou, Zhejiang, People's Republic of China. ${ }^{2}$ Department of Nursing, Huzhou Third Municipal Hospital, 2088 East Tiaoxi Rd, Huzhou, Zhejiang, People's Republic of China.

${ }^{3}$ Medical College of Nursing, Huzhou University, No. 759 Erhuan East Road, Huzhou 313000, Zhejiang, China. ${ }^{4}$ Graduate School of Medicine Faculty, Zhejiang University, No. 866 Yuhangtang Road, Xihu, Hangzhou 310058, Zhejiang, People's Republic of China. ${ }^{5}$ Department of Oncology, Huzhou Central Hospital, Affiliated Central Hospital Huzhou University, No. 1558, Sanhuan North Road, Wuxing, Huzhou 313000, Zhejiang, China.

Received: 24 March 2021 Accepted: 11 May 2021

Published online: 26 May 2021

\section{References}

Bensinger SJ, Christofk HR. New aspects of the Warburg effect in cancer cell biology. Semin Cell Dev Biol. 2012;23(4):352-61.

Bin Y, Xiaodi T, Zhixin W, et al. TUG1 promotes prostate cancer progression by acting as a ceRNA of miR-26a. 2018. Biosci Rep. https://doi.org/10.1042/ BSR20180677.

Bing Z, Huilin Ye, Jianming C, et al. LncRNA TUG1 sponges miR-145 to promote cancer progression and regulate glutamine metabolism via Sirt3/GDH axis. Oncotarget. 2017:8:113650-61.

Caihui Xu, Yinmou G, Haiyan L, et al. TUG1 confers cisplatin resistance in esophageal squamous cell carcinoma by epigenetically suppressing PDCD4 expression via EZH2. Cell Biosci. 2018;8:61.

Chuan He, Zhigang L, Li J, et al. IncRNA TUG1-mediated Mir-142-3p downregulation contributes to metastasis and the epithelial-to-mesenchymal transition of hepatocellular carcinoma by targeting ZEB1. Cell Physiol Biochem. 2018;48:1928-41.

Chu-Hai X, Yan-Ming C, Yan H, et al. Long non-coding RNA TUG1 contributes to tumorigenesis of human osteosarcoma by sponging miR-9-5p and regulating POU2F1 expression. Tumour Biol. 2016;37:15031-41.

Chuhai X, Binwei C, Boyi W, et al. LncRNA TUG1 promotes cell proliferation and suppresses apoptosis in osteosarcoma by regulating miR-212-3p/ FOXA1 axis. Biomed Pharmacother. 2018;97:1645-53.

Claudia S, Greta C, Paola B, et al. Progress in cancer mortality, incidence, and survival: a global overview. Eur J Cancer Prev. 2020;29(5):367-81.

Daniela C, Rosario A, Giovanni C, et al. Cholesterol homeostasis modulates platinum sensitivity in human ovarian cancer. Cells. 2020;9:828.

Dianhui X, Lin L, Min C, et al. Knockdown of IncRNA TUG1 enhances radiosensitivity of prostate cancer via the TUG1/miR-139-5p/SMC1A axis. OncoTargets Ther. 2020;13:2319-31.

Ding Y, Chan EC, Liu L, et al. Long noncoding RNAs: important participants and potential therapeutic targets for myocardial ischaemia reperfusion injury. Clin Exp Pharm Physiol. 2020. https://doi.org/10.1111/1440-1681. 13375.

Dong LV, Ying X, Qi Y, et al. Long non-coding RNA TUG1 promotes cell proliferation and inhibits cell apoptosis, autophagy in clear cell renal cell carcinoma via MiR-31-5p/FLOT1 axis. OncoTargets Ther. 2020;13:5857-68.

Fan S, Yang Z, Ke Z, et al. Downregulation of the long non-coding RNA TUG1 is associated with cell proliferation, migration, and invasion in breast cancer. Biomed Pharmacother. 2017;95:1636-43. 
Farbod E, Mohammad T, Davood OM, et al. Expression of long non-coding RNAs (IncRNAs) has been dysregulated in non-small cell lung cancer tissues. BMC Cancer. 2019;19:222.

Farhad I, Rebecca LS, Ahmedin J. The changing landscape of cancer in the USA - opportunities for advancing prevention and treatment. Nature reviews. Clin Oncol. 2020;17(10):631-49.

Feraydoon A, Soudeh G-F, Mohammad T, et al. Expression analysis of a panel of long non-coding RNAs (IncRNAs) revealed their potential as diagnostic biomarkers in bladder cancer. Genomics. 2020;112:677-82.

Fu-Bing Y, Juan S, Jia-Man Y, et al. MiR-19a-3p regulates the Forkhead box F2-mediated Wnt/ $\beta$-catenin signaling pathway and affects the biological functions of colorectal cancer cells. World J Gastroenterol. 2020;26:627-44

Gan Yu, Hui Z, Weimin Y, et al. IncRNA TUG1 promotes cisplatin resistance by regulating CCND2 via epigenetically silencing miR-194-5p in bladder cancer. Mol Ther Nucleic Acids. 2019;16:257-71.

Gang L, Keyu L, Xinhui D. Long non-coding RNA TUG1 promotes proliferation and inhibits apoptosis of osteosarcoma cells by sponging miR-132-3p and upregulating SOX4 expression. Yonsei Med J. 2018;59:226-35.

Gang Li, Peiming Z, Huiling W, et al. Long non-coding RNA TUG1 modulates proliferation, migration, and invasion of acute myeloid leukemia cells via regulating miR-370-3p/MAPK1/ERK. OncoTargets Ther. 2019;12:10375-88.

Gang Li, Jie Y, Tie C, et al. TUG1 knockdown inhibits the tumorigenesis and progression of prostate cancer by regulating microRNA-496/Wnt/ $\beta$-catenin pathway. Anticancer Drugs. 2020;31:592-600.

Guttman M, John LR. Modular regulatory principles of large non-coding RNAs. Nature. 2012;482(7385):339-46.

Hao S-D, Ma J-X, Liu Y, et al. Long non-coding TUG1 accelerates prostate cancer progression through regulating miR-128-3p/YES1 axis. Eur Rev Med Pharmacol Sci. 2020;24:619-32.

He Pan Xu, Yong-Qiang W-J, et al. LncRNA LINC00210 regulated radiosensitivity of osteosarcoma cells via miR-342-3p/GFRA1 axis. J Clin Lab Anal. 2020;34:e23540

Heng Li, Guofeng T, Feipeng T, et al. Long non-coding RNA TUG1 promotes osteosarcoma cell proliferation and invasion through inhibition of microRNA-212-3p expression. Exp Ther Med. 2018;16:779-87.

Hui Bingqing Xu, Yetao ZB, et al. TUG1Overexpressed long noncoding RNA affects the cell cycle, proliferation, and apoptosis of pancreatic cancer partly through suppressing RND3 and MT2A. Onco Targets Ther. 2019;12:1043-57.

Hui-Yuan Z, Ming-Hua S, Xiao Y, et al. Overexpression of long non-coding RNA TUG1 promotes colon cancer progression. Med Sci Monit. 2016:22:3281-7.

Jiang Huijuan Hu, Xigang ZH, et al. Down-regulation of LncRNA TUG1 enhances radiosensitivity in bladder cancer via suppressing HMGB1 expression. Radiat Oncol. 2017;12:65.

Jin G, Yang Y, Tuo G, et al. LncRNA TUG1 promotes tumor growth and metastasis of esophageal squamous cell carcinoma by regulating XBP1 via competitively binding to miR-498. Neoplasma. 2020;67:751-61.

Ji-Yu B, Ben J, Jian-Bin Ma, et al. HOTAIR and androgen receptor synergistically increase GLI2 transcription to promote tumor angiogenesis and cancer stemness in renal cell carcinoma. Cancer Lett. 2021:498:70-9.

Jun Li, Meng Z, Gang An, et al. LncRNA TUG1 acts as a tumor suppressor in human glioma by promoting cell apoptosis. Exp Biol Med (maywood). 2016a;241:644-9.

Jun Li, Gang An, Meng Z, et al. Long non-coding RNA TUG1 acts as a miR26a sponge in human glioma cells. Biochem Biophys Res Commun. 2016b;477:743-8

Jun Q, Hongxia B, Hong Li. Correlation of long non-coding RNA taurineupregulated gene 1 with disease conditions and prognosis, as well as its effect on cell activities in acute myeloid leukemia. Cancer Biomark. 2018a;23:569-77.

Jun Lv, Yongkui K, Zhiqiang G, et al. LncRNA TUG1 interacting with miR-144 contributes to proliferation, migration and tumorigenesis through activating the JAK2/STAT3 pathway in hepatocellular carcinoma. Int J Biochem Cell Biol. 2018b;101:19-28.

Ke Xu, Lianfeng Z. Inhibition of TUG1/miRNA-299-3p axis represses pancreatic cancer malignant progression via suppression of the Notch1 pathway. Dig Dis Sci. 2020;65:1748-60.
Ke Z, Hong Z, Bo Y, et al. TUG1/miR-133b/CXCR4 axis regulates cisplatin resistance in human tongue squamous cell carcinoma. Cancer Cell Int. 2020;20:148.

Kewei R, Zhen Li, Yahua Li, et al. Long noncoding RNA taurine-upregulated gene 1 promotes cell proliferation and invasion in gastric cancer via negatively modulating miRNA-145-5p. Oncol Res. 2017;25:789-98.

Kunkun S, Yan Li. LncRNA TUG1 promotes the development of osteosarcoma through RUNX2. Exp Ther Med. 2019;18:3002-8.

Lei T, Zhi-Feng Z, Ling X, et al. Taurine up-regulated 1 accelerates tumorigenesis of colon cancer by regulating miR-26a-5p/MMP14/p38 MAPK/Hsp27 axis in vitro and in vivo. Life Sci. 2019;239:117035.

Li L, Gan Z-H, Qin L, et al. AlB1 regulates the ovarian cancer cell cycle through TUG1. Eur Rev Med Pharmacol Sci. 2017a;21:5610-7.

Li ZQ, Zou R, Ouyang KX, et al. An in vitro study of the long non-coding RNA TUG1 in tongue squamous cell carcinoma. J Oral Pathol Med. 2017b;46(10):956-60.

Li Q, Song W, Wang J. TUG1 confers Adriamycin resistance in acute myeloid leukemia by epigenetically suppressing miR-34a expression via EZH2. Biomed Pharmacother. 2019;109:1793-801.

Liang W, Zhenxian Z, Weidong F, et al. Long non-coding RNA TUG1 promotes colorectal cancer metastasis via EMT pathway. Oncotarget. 2016;7:51713-9.

Liang Z, Hongwei S, Hongru K, et al. The Lncrna-TUG1/EZH2 axis promotes pancreatic cancer cell proliferation, migration and EMT phenotype formation through sponging Mir-382. Cell Physiol Biochem. 2017:42:2145-58.

Li-Jun D, Min D, Li-Jun H, et al. Long noncoding RNA TUG1 alleviates extracellular matrix accumulation via mediating microRNA-377 targeting of PPARy in diabetic nephropathy. Biochem Biophys Res Commun. 2017:484:598-604.

Lin J, Wenchao W, Guoli Li, et al. High TUG1 expression is associated with chemotherapy resistance and poor prognosis in esophageal squamous cell carcinoma. Cancer Chemother Pharmacol. 2016;78:333-9.

Lin YH, Wu MH, Huang YH, et al. TUG1 is a regulator of AFP and serves as prognostic marker in non-hepatitis B non-hepatitis $C$ hepatocellular carcinoma. Cells. 2020;9(2):262.

Lize Gu, Qing Li, Hao L, et al. Long noncoding RNA TUG1 promotes autophagy-associated paclitaxel resistance by sponging miR-29b-3p in ovarian cancer cells. OncoTargets Ther. 2020;13:2007-19.

Luo Wenfeng Y, Huilan ZX, et al. Long non-coding RNA taurine-upregulated gene 1 correlates with unfavorable prognosis in patients with refractory or relapsed acute myeloid leukemia treated by purine analogue based chemotherapy regimens. Cancer Biomark. 2018;23:485-94.

Mai M, Safinaz I, Olfat S, et al. Serum expression and diagnostic potential. of long non-coding RNAs NEAT1 and TUG1 in viral hepatitis $C$ and viral hepatitis C-associated hepatocellular carcinoma. Clin Biochem. 2020;84:38-44.

Miaomiao G, Xun W, Lin M, et al. Steroid receptor coactivator-1 enhances the stemness of glioblastoma by activating long noncoding RNA XIST/miR152/KLF4 pathway. Cancer Sci. 2021;112:604-18.

Mingjun F, Chunyan Li, Pengjuan He, et al. Knockdown of long noncoding RNA-taurine-upregulated gene 1 inhibits tumor angiogenesis in ovarian cancer by regulating leucine-rich a-2-glycoprotein-1. Anticancer Drugs. 2019;30:562-70.

Mingzhu Z, Wanting F, Li W, et al. LncRNA TUG1 promotes esophageal cancer development through regulating PLK1 expression by sponging miR1294. Biotechnol Lett. 2020;42:2537-49.

Nashwa E-K, Fathy MH, Mohamed Y, et al. Tissue-based long non-coding RNAs "PVT1, TUG1 and MEG3" signature predicts cisplatin resistance in ovarian cancer. Genomics. 2020;112:4640-6.

Ozcan Selahattin C, Aybike S, Tugba SH, et al. PFKFB2 regulates glycolysis and proliferation in pancreatic cancer cells. Mol Cell Biochem. 2020;470:115-29.

Pan W, Zhuanbo $Y$, Ting Ye, et al. IncTUG1/miR-144-3p affect the radiosensitivity of esophageal squamous cell carcinoma by competitively regulating c-MET. J Exp Clin Cancer Res. 2020;39:7.

Pei-Chin L, Hsien-Da H, Chun-Chi C, et al. Long noncoding RNA TUG1 is downregulated in non-small cell lung cancer and can regulate CELF1 on binding to PRC2. BMC Cancer. 2016;16:583.

Peng K, Guihua J, Qingyang M, et al. Long non-coding RNA taurine-upregulated gene 1 promotes cells proliferation, migration and invasion while 
represses apoptosis, and upregulates AURKA expression in hepatocellular carcinoma. Int J Clin Exp Pathol. 2018a;11:3199-207.

Peng G, Guohui Z, Jialin M, et al. Upregulation of long noncoding RNA TUG1 promotes bladder cancer cell proliferation, migration, and invasion by inhibiting miR-29c. Oncol Res. 2018b;26:1083-91.

Protopsaltis NJ, Wei L, Eric N, et al. Interleukin-22 promotes tumor angiogenesis. Angiogenesis. 2019;22:311-23.

Qian L, Hui L, Hepeng C, et al. Downregulation of long noncoding RNA TUG1 inhibits proliferation and induces apoptosis through the TUG1/miR-142/ZEB2 axis in bladder cancer cells. OncoTargets Ther. 2017:10:2461-71.

Qingqing Y, Xianjuan S, Xiaopeng C, et al. Elevated serum IncRNA TUG1 levels are a potential diagnostic biomarker of multiple myeloma. Exp Hematol. 2019;79:47-55.e2.

Qun D, Jingyi D, Jinrong Z, et al. Long non-coding RNA TUG1 promotes cell progression in hepatocellular carcinoma via regulating miR-216b-5p/ DLX2 axis. Cancer Cell Int. 2020;20:8.

Qunli W, Qi C. Role of taurine upregulated gene 1 as a predictor of poor outcome in osteosarcoma. J Cancer Res Ther. 2018;14:S405-9.

Shan L, Yantong Y, Weiwei W, et al. Long noncoding RNA TUG1 promotes cell proliferation and migration of renal cell carcinoma via regulation of YAP. J Cell Biochem. 2018;119:9694-706.

Shen X, Hu X, Mao J, et al. The long noncoding RNA TUG1 is required for TGF- $\beta /$ TWIST1/EMT-mediated metastasis in colorectal cancer cells. Cell Death Dis. 2020;11(1):65.

Shenfa Z, Fengxian L, Pingping W. Upregulation of long noncoding RNA TUG1 contributes to the development of laryngocarcinoma by targeting miR145-5p/ROCK1 axis. J Cell Biochem. 2019;120:13392-402.

Shuang L, Songzi Z, Peng W, et al. LncRNA, TUG1 regulates the oral squamous cell carcinoma progression possibly via interacting with $\mathrm{Wnt} / \beta$-catenin signaling. Gene. 2017;608:49-57.

Sisi W, Shiping S, Xinliang Z, et al. SNHG5 inhibits the progression of EMT through the ubiquitin-degradation of MTA2 in oesophageal cancer. Carcinogenesis. 2021;42:315-26.

Taft RJ, Pang KC, Mercer TR, et al. Non-coding RNAs: regulators of disease. J Pathol. 2010;220(2):126-39.

Tang L-P, Ding J-B, Liu Z-H, et al. LncRNA TUG1 promotes osteoarthritisinduced degradation of chondrocyte extracellular matrix via miR-195/ MMP-13 axis. Eur Rev Med Pharmacol Sci. 2018;22:8574-81.

Tonghuai Li, Yan C, Jingjing Z, et al. LncRNA TUG1 promotes cells proliferation and inhibits cells apoptosis through regulating AURKA in epithelial ovarian cancer cells. Medicine (baltimore). 2018:97:e12131.

Tong-Huai Li, Jing-Jing Z, Shao-Xiao L, et al. Long non-coding RNA taurineupregulated gene 1 predicts unfavorable prognosis, promotes cells proliferation, and inhibits cells apoptosis in epithelial ovarian cancer. Medicine (baltimore). 2018;97:e0575.

Tongzhou H, Zhenghua F, Huafang S, et al. Polydatin inhibits proliferation and promotes apoptosis of doxorubicin-resistant osteosarcoma through LncRNA TUG1 mediated suppression of Akt signaling. Toxicol Appl Pharmacol. 2019;371:55-62.

Wang Heping Yu, Yanzhang FS, et al. Knockdown of long noncoding RNA TUG1 inhibits the proliferation and cellular invasion of osteosarcoma cells by sponging miR-153. Oncol Res. 2018;26:665-73.

Wei Q, Zhengting R, Xueguan L. Knockdown of long non-coding RNA TUG 1 suppresses nasopharyngeal carcinoma progression by inhibiting epithelial-mesenchymal transition (EMT) via the promotion of miR-384. Biochem Biophys Res Commun. 2019;509:56-63.

Wei Z, Xue J, Shuxia Y. COL1A1 IncRNA tug1 promotes cell proliferation, migration, and invasion in hepatocellular carcinoma via regulating miR29c-3p/ axis. Cancer Manag Res. 2020;12:6837-47.

Weide Z, Yuhua L, Jing Z, et al. Long non-coding RNA taurine upregulated gene 1 targets miR-185 to regulate cell proliferation and glycolysis in acute myeloid leukemia cells in vitro. OncoTargets Ther. 2020:13:7887-96.

Xia Li, Sheng-Wu W, Xi-Ling Li, et al. Knockdown of long non-coding RNA TUG1 depresses apoptosis of hippocampal neurons in Alzheimer's disease by elevating microRNA-15a and repressing ROCK1 expression. Inflamm Res. 2020:69:897-910.

Xiao G, Qingwei W, Ying W, et al. The REG Y inhibitor NIP30 increases sensitivity to chemotherapy in p53-deficient tumor cells. Nat Commun. 2020:11:3904
Xiao-Bo Z, Guo-Sheng R. LncRNA taurine-upregulated gene 1 promotes cell proliferation by inhibiting microRNA-9 in MCF-7 cells. J Breast Cancer. 2016:19:349-57.

Xiaoqiang W, Xiaomin B, Zhonghui Y, et al. The IncRNA TUG1 promotes cell growth and migration in colorectal cancer via the TUG1-miR-145-5pTRPC6 pathway. Biochem Cell Biol. 2021;99:249-60.

Xiaoqiu Y, Nana X, Hai-Jun Q, et al. Long noncoding RNA TUG1 facilitates cell ovarian cancer progression through targeting MiR-29b-3p/MDM2 axis. Anat Rec (hoboken). 2020:303:3024-34.

Xinfeng W, Lina Z, Fan Z, et al. Long non-coding RNA taurine-upregulated gene 1 correlates with poor prognosis, induces cell proliferation, and represses cell apoptosis via targeting aurora kinase A in adult acute myeloid leukemia. Ann Hematol. 2018;97:1375-89.

Xiong Z-J, Zhang Q, Wang D-X, et al. Overexpression of TUG1 promotes neuronal death after cerebral infarction by regulating microRNA-9. Eur Rev Med Pharmacol Sci. 2018:22:7393-400

Xiufu $\mathrm{H}$, Yanming Y, Yongjie S, et al. LncRNA TUG1 affects cell viability by regulating glycolysis in osteosarcoma cells. Gene. 2018;674:87-92.

Xu Y, Wang J, Qiu M, et al. Upregulation of the long noncoding RNA TUG1 promotes proliferation and migration of esophageal squamous cell carcinoma. Tumour Biol. 2015;36(3):1643-51.

Xuemin W, Yuehua Z, Jin Q, et al. Low expression of TUG1 promotes cisplatin sensitivity in cervical cancer by activating the MAPK pathway. J BUON. 2019;24:1020-6.

Yang Li, Tao Z, Yanhui Z, et al. Targeting the FOXM1-regulated long noncoding RNA TUG1 in osteosarcoma. Cancer Sci. 2018a;109:3093-104.

Yang Y, De-Ming S, Jun-Feng Y, et al. Long noncoding RNA TUG1 promotes renal cell carcinoma cell proliferation, migration and invasion by downregulating microRNA-196a. Mol Med Rep. 2018b;18:5791-8.

Yao R-W, Wang Y, Chen L-L. Cellular functions of long noncoding rnas. Nat Cell Biol. 2019:21(5):542.

Yebin Lu, Ling T, Zhipeng Z, et al. Long noncoding RNA TUG1/miR-29c axis affects cell proliferation, invasion, and migration in human pancreatic cancer. Dis Markers. 2018;2018:6857042.

Yihui Li, Chengting D, Yi Y, et al. The mechanisms of IncRNA Tug1 in islet dysfunction in a mouse model of intrauterine growth retardation. Cell Biochem Funct. 2020;38:1129-38.

Yin T, Ping Y, Yunfeng Z, et al. LncRNA TUG1 contributes to ESCC progression via regulating miR-148a-3p/MCL-1/Wnt/ $\beta$-catenin axis in vitro. Thorac Cancer. 2020;11:82-94.

Yingying Hu, Xiangwei S, Chenchen M, et al. Upregulation of long noncoding RNA TUG1 promotes cervical cancer cell proliferation and migration. Cancer Med. 2017:6:471-82.

Yong W, Tao Y, Zhen Z, et al. Long non-coding RNA TUG1 promotes migration and invasion by acting as a ceRNA of miR-335-5p in osteosarcoma cells. Cancer Sci. 2017;108:859-67.

Young TL, Matsuda T, Cepko CL. The noncoding RNA taurine upregulated gene 1 is required for differentiation of the murine retina. Curr Biol. 2005:15:501-12.

Yu Xiao Hu, Lei LS, et al. Long non-coding RNA Taurine upregulated gene 1 promotes osteosarcoma cell metastasis by mediating HIF-1a via miR143-5p. Cell Death Dis. 2019;10:280.

Yuan HW, Wen GL, Jun L, et al. LncRNA TUG1 promotes growth and metastasis of cholangiocarcinoma cells by inhibiting miR-29a. Cancer Manag Res. 2020;12:11103-11.

Yunsheng Li, Dan Z, Liutong P, et al. Knockdown of TUG1 by shRNA inhibited renal cell carcinoma formation by miR-299-3p/VEGF axis in vitro and in vivo. Eur J Pharmacol. 2019:860:172536.

Yuqing P, Kexin Li, Xiaoying L, et al. miR-1299/NOTCH3/TUG1 feedback loop contributes to the malignant proliferation of ovarian cancer. Oncol Rep. 2020;44:438-48.

Zhang $E$, Yin D, Sun M, et al. P53-regulated long non-coding RNA TUG1 affects cell proliferation in human non-small cell lung cancer, partly through epigenetically regulating HOXB7 expression. Cell Death Dis. 2014;5:1243.

Zhang Z, Wang X, Cao S, et al. The long noncoding RNA TUG1 promotes laryngeal cancer proliferation and migration. Cell Physiol Biochem. 2018:49:2511-20.

Zhang B, Sun Y-F, Zhang X-M, et al. TUG1 weakens the sensitivity of acute myeloid leukemia cells to cytarabine by regulating miR-655-3p/CCND1 axis. Eur Rev Med Pharmacol Sci. 2020;24:4940-53. 
Zhao Z, Wang B, Hao J, et al. Downregulation of the long non-coding RNA taurine-upregulated gene 1 inhibits glioma cell proliferation and invasion and promotes apoptosis. Oncol Lett. 2018;15(3):4026-32.

Zhao Z-Y, Zhao Y-C, Liu W. Long non-coding RNA TUG1 regulates the progression and metastasis of osteosarcoma cells via miR-140-5p/PFN2 axis. Eur Rev Med Pharmacol Sci. 2019;23:9781-92.

Zhenghua Z, Ran X, Caiwei Li, et al. LncRNA TUG1 promotes cisplatin resistance in esophageal squamous cell carcinoma cells by regulating $\mathrm{Nrf2}$. Acta Biochim Biophys Sin (shanghai). 2019:51:826-33.

Zhenqiang W, Xinjing W, Tianqi Z, et al. LncRNA MALAT1 promotes gastric cancer progression via inhibiting autophagic flux and inducing fibroblast activation. Cell Death Dis. 2021;12:368.

Zhifeng W, Jingmei L, Rong W, et al. Long non-coding RNA taurine upregulated gene 1 (TUG1) downregulation constrains cell proliferation and invasion through regulating cell division cycle 42 (CDC42) expression via MiR-498 in esophageal squamous cell carcinoma cells. Med Sci Monit. 2020;26:e919714.
Zhiqiang Y, Zhibin P, Zhichao $W$, et al. The oncogenic role of REG $\gamma$ is exerted by activating the $\mathrm{Wnt} / \beta$-catenin signaling pathway in osteosarcoma. Am J Transl Res. 2020;12:563-73.

Zhou Qiang Hu, Yuan TX. Anticancer potential of TUG1 knockdown in cisplatin-resistant osteosarcoma through inhibition of MET/Akt signalling. J Drug Target. 2020;28:204-11.

Zhulei S, Gui H, Hepeng C. Nrf2Transcription factor induces the up-regulation of IncRNA to promote progression and adriamycin resistance in urothelial carcinoma of the bladder. Cancer Manag Res. 2019;11:6079-90.

\section{Publisher's Note}

Springer Nature remains neutral with regard to jurisdictional claims in published maps and institutional affiliations.
Ready to submit your research? Choose BMC and benefit from:

- fast, convenient online submission

- thorough peer review by experienced researchers in your field

- rapid publication on acceptance

- support for research data, including large and complex data types

- gold Open Access which fosters wider collaboration and increased citations

- maximum visibility for your research: over $100 \mathrm{M}$ website views per year

At BMC, research is always in progress.

Learn more biomedcentral.com/submissions 